\title{
Junction edge passivation study of silicon surface barrier detectors using organic films deposited by L-B technique
}

\author{
A RAY ${ }^{1,2, *}$, SIPRA CHOUDHURY ${ }^{3}$, VISHAL SINGH ${ }^{4}$, C A BETTY ${ }^{2,3}$ \\ and T V CHANDRASEKHAR RAO ${ }^{1,2}$ \\ ${ }^{1}$ Technical Physics Division, Bhabha Atomic Research Centre, Trombay, Mumbai 400 085, India \\ ${ }^{2}$ Homi Bhabha National Institute, Anushakti Nagar, Mumbai 400 094, India \\ ${ }^{3}$ Chemistry Division, Bhabha Atomic Research Centre, Trombay, Mumbai 400 085, India \\ ${ }^{4}$ Material Science Division, Bhabha Atomic Research Centre, Trombay, Mumbai 400 085, India \\ *Author for correspondence (array@barc.gov.in)
}

MS received 16 October 2018; accepted 25 February 2019

\begin{abstract}
A new technique to passivate silicon surfaces using $\mathrm{SnO}_{2}$ films, produced by decomposing organic films of the octadecylamine-stannate complex, deposited by the Langmuir-Blodgett (L-B) technique, has been attempted to fabricate silicon surface barrier detectors. This method of passivation is relatively simpler and can be carried out at a much lower temperature as compared to the usual passivation method of the silicon surface by growing the $\mathrm{SiO}_{2}$ layer on it. Also, the passivating layer of $\mathrm{SnO}_{2}$ produced in this new method has a good shelf-life. The detectors fabricated with a passivating layer of $\mathrm{SnO}_{2}$ were subsequently tested for $I-V$, alpha spectrum and long-term performance.
\end{abstract}

Keywords. Tin oxide; nanoparticles; passivation; surface-barrier; detector.

\section{Introduction}

The controlled transfer of organized monolayers of amphiphilic molecules onto a solid substrate from the airwater interface was the first molecular-scale technology for the development of new materials. Various aspects of Langmuir-Blodgett (L-B) films, in greater detail, were discussed by Zasadzinski et al [1]. Although one can come across lots of applications of the L-B deposition method in the literature, very little information is available with respect to the application of the L-B deposition in nuclear detector development. With regard to the passivation of silicon detectors, silicon dioxide-passivated Si-detectors [2,3] are known for their long-term stability. Organic epoxy-passivated silicon surface barrier (SSB) detectors [4-7] are also widely used by researchers. In SSB detectors, organic epoxy-based passivation of the $\mathrm{Si}$-surface is carried out at room temperature by using two-component epoxy. The right quantity of an aminebased hardener is used to properly cure epoxy resin which in turn results in very low leakage current and stable detector performance. The epoxy used in these 'epoxy passivated detectors' is of hydrophilic nature. As a result of this, the epoxy reacts with atmospheric moisture as well as oxygen and deteriorates with time. Accordingly, the reverse leakage currents of the fabricated detectors increase with time. Thus, to enhance the longevity of these detectors, one needs to look for a passivating layer based on some new material. In this work, an amine-based organic film (viz. the octadecylaminestannate complex, i.e., the ODA-stannate complex) was used as a precursor to grow tin oxide via the organic route and was used as a passivating layer of good shelf life for SSB detectors. This film was formed as a result of the decomposition of an amine-based L-B film by heating it at a temperature of $\sim 600^{\circ} \mathrm{C}$. Another method of passivation, viz., oxidizing silicon to get a good quality thin $\mathrm{SiO}_{2}$ layer (breakdown voltage: $\left.7 \mathrm{MV} \mathrm{cm}^{-1}\right)$ requires a high temperature $\left(1000-1200^{\circ} \mathrm{C}\right)$ furnace, high quality oxygen, etc. In contrast, growing the $\mathrm{SnO}_{2}$ film $(\rho \approx 10-20 \mathrm{M} \Omega \mathrm{cm})$ on a silicon wafer is a new as well as a simpler technique [8,9] which has been explored to study the passivation of silicon detectors. All these factors, i.e., good shelf life, simpler fabrication methodology, accurate thickness control and a fabrication process involving a lower thermal budget are the advantages available to a researcher to explore the tin oxide passivation study. It is carried out by initially depositing the ODA-stannate complex on silicon wafer edges with the help of the L-B deposition technique, followed by heating the sample in a furnace in air at a relatively low temperature $\left(600^{\circ} \mathrm{C}\right)$ to decompose the L-B layer to form a $\mathrm{SnO}_{2}$ film on silicon. Tin oxide grown under the above conditions consists of $\mathrm{SnO}_{2}$ nanoparticles. The layer behaves like a depletion layer and offers very high resistance. The L-B method was chosen to get a very uniform film [8] of the ODA-stannate complex. This film is the precursor which after heating to a temperature of $600^{\circ} \mathrm{C}$, decomposed into a uniform tin oxide film. For 95-100 monolayers of the ODAstannate complex, the thickness of the tin oxide film will be $\sim 200 \mathrm{~nm}[10]$. So, in this method, one can have an idea about the thickness of the newly produced tin oxide film from 

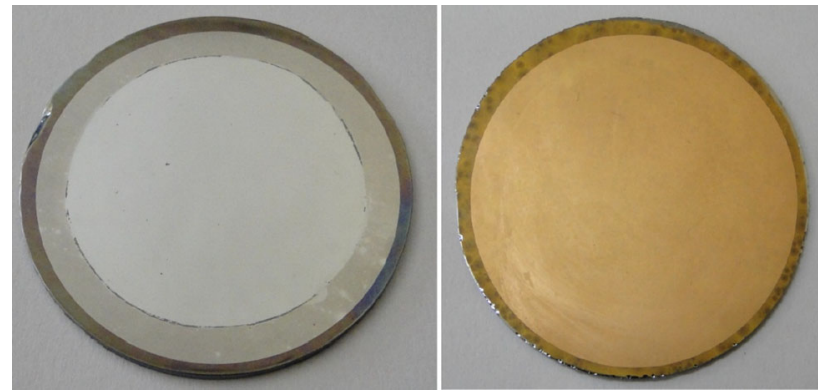

Figure 1. Front and back side photographs of an unmounted detector.

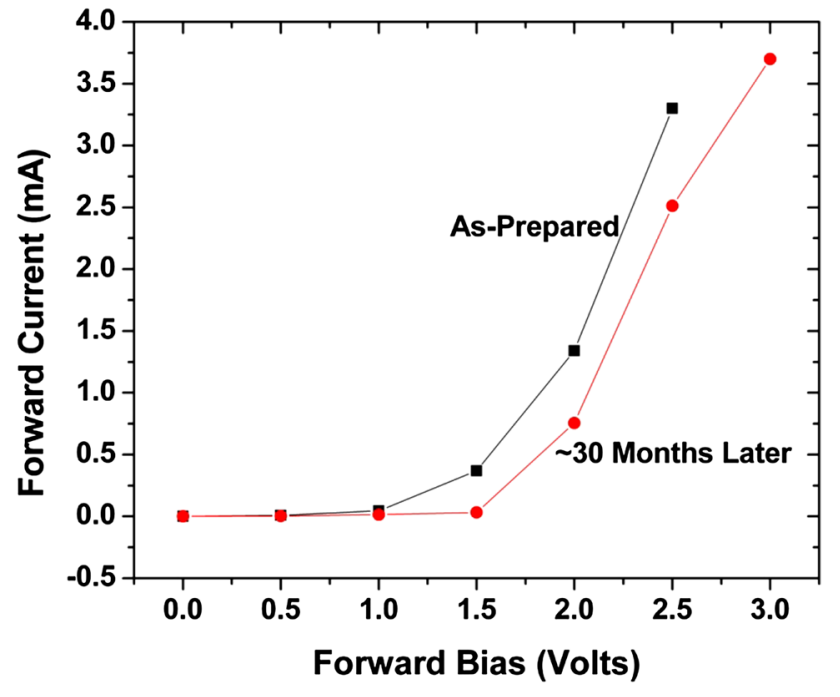

Figure 2. Forward characteristics.

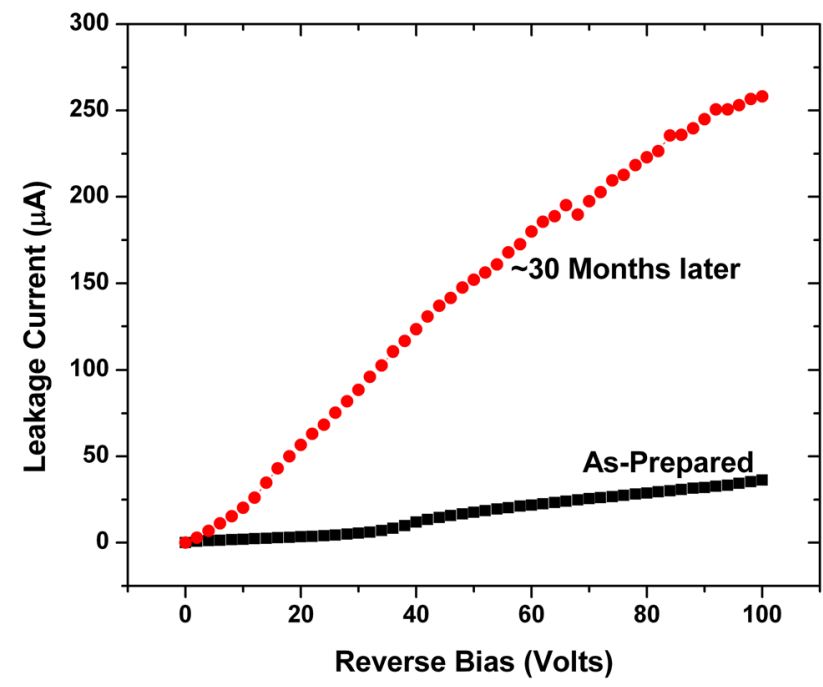

Figure 3. Reverse characteristics.

the deposited number of layers. On the other hand, though spin coating, dip coating, etc. are relatively simpler methods, neither can they produce films of good uniformity nor can they achieve an accurate thickness control.

\section{Detector fabrication}

P-type silicon wafers $\left(2^{\prime \prime}\right.$ diameter, $1 \mathrm{~mm}$ thickness and $8 \mathrm{k} \Omega \mathrm{cm}$ resistivity) with their front side chemically polished and backside coated with gold by electroless plating were taken as the starting material. Their chemically polished front sides were partly covered with tape (as mask) with a diameter of $38 \mathrm{~mm}$ at the central part leaving the peripheral annular region (at the edge) open. Their backside gold layers were fully covered by tape. Multilayer L-B films of the ODA-stannate complex (95-100 layers) were deposited on the unmasked part of the silicon wafers. The detailed procedure for deposition is explained by Choudhury et al [8].

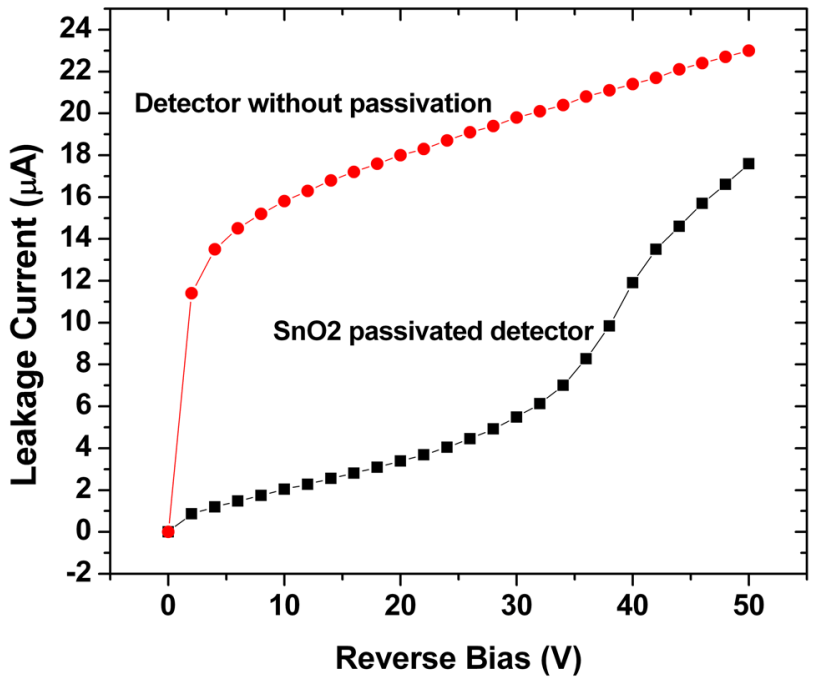

Figure 4. Comparative reverse $I-V$ characteristics of the detector with/without passivation. Graph marked with 'Detector without passivation' is reprinted from [11], copyright (2004), with permission from Allied Publishers Pvt. Ltd., New Delhi, India.

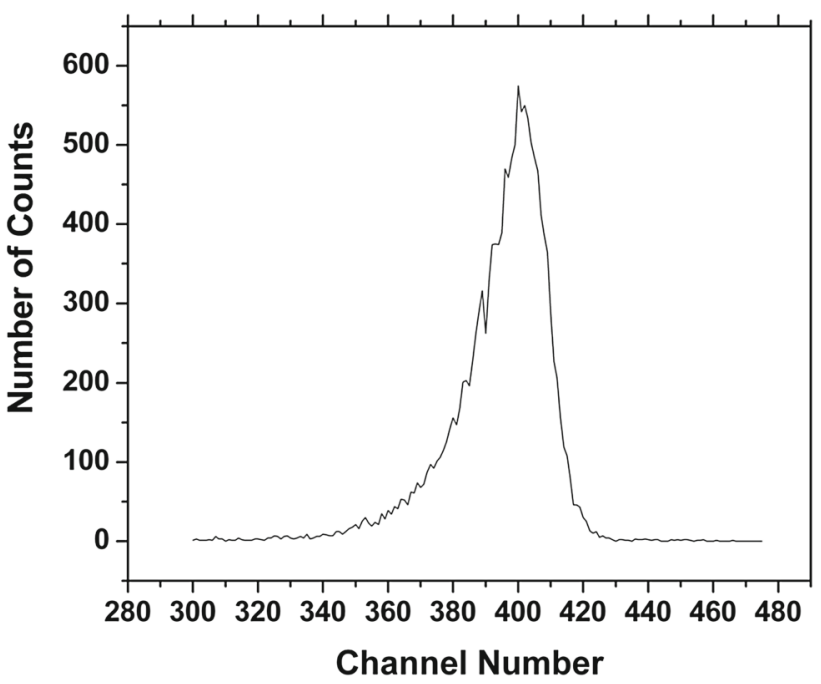

Figure 5. Alpha spectrum. 
After deposition, the tapes used for masking were removed and the covered portions under the masks were thoroughly cleaned using trichloroethylene. Then, these samples were kept in a clean quartz tube and put in a furnace (one sample at a time), heated with a ramping rate of $5^{\circ} \mathrm{C} \min ^{-1}$ (approx.) to reach $600^{\circ} \mathrm{C}$ (in the presence of air) for $2 \mathrm{~h}$ and held at that temperature for another $3 \mathrm{~h}$. Due to this temperature, the multilayer L-B films decomposed to form $\mathrm{SnO}_{2}$ films at the edges of the wafers. An aluminium film with a thickness of $40 \mu \mathrm{g} \mathrm{cm}^{-2}$ was evaporated onto the central part of the

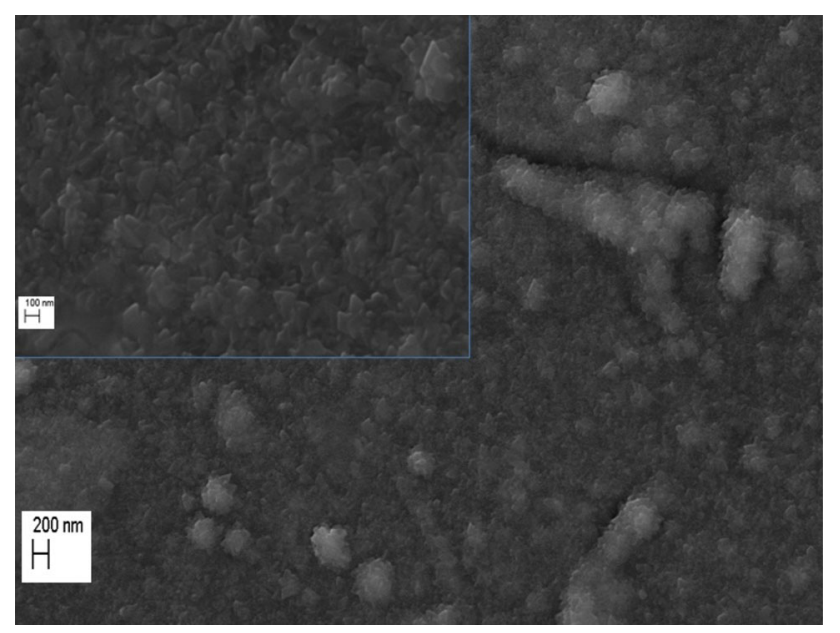

Figure 6. SEM image of the $\mathrm{SnO}_{2}$ surface. polished side partly covering the $\mathrm{SnO}_{2}$ layer. Subsequently, a gold film with a thickness of $40 \mu \mathrm{g} \mathrm{cm}^{-2}$ was evaporated on the backside.

\section{Test results}

The detectors were tested for the $I-V$ (forward and reverse) characteristics and the alpha spectrum. Figure 1 shows the front and back side of one of the fabricated detectors before mounting and encapsulation. The tin oxide thin film is visible at the edge on the front side on which aluminium metallization was performed to make the $\mathrm{Al} / \mathrm{p}$-Si Schottky barrier junction. Figures 2 and 3 show forward and reverse characteristics of the detector, respectively. To have an idea about long-term performance, both the 'as-prepared' and '30 months later' characteristics have been measured and incorporated in forward as well as in reverse characteristics.

The greater value of the leakage current is due to the larger active area (diameter of active area $\sim 38 \mathrm{~mm}$ ) of the surface barrier detector. Nevertheless, the effectiveness of the tin-oxide passivating layer is clearly shown in figure 4 by a comparison of the reverse $I-V$ characteristics of a tinoxide passivated detector with those of a detector without any passivating layer. The reverse characteristics of the 'detector without any passivation' are reprinted from the Proceedings of DAE Solid State Physics Symposium 2003, for comparison purposes [11]. The alpha spectrum of the passivated detector obtained using the $\mathrm{Am}-\mathrm{Pu}$ alpha source is shown in

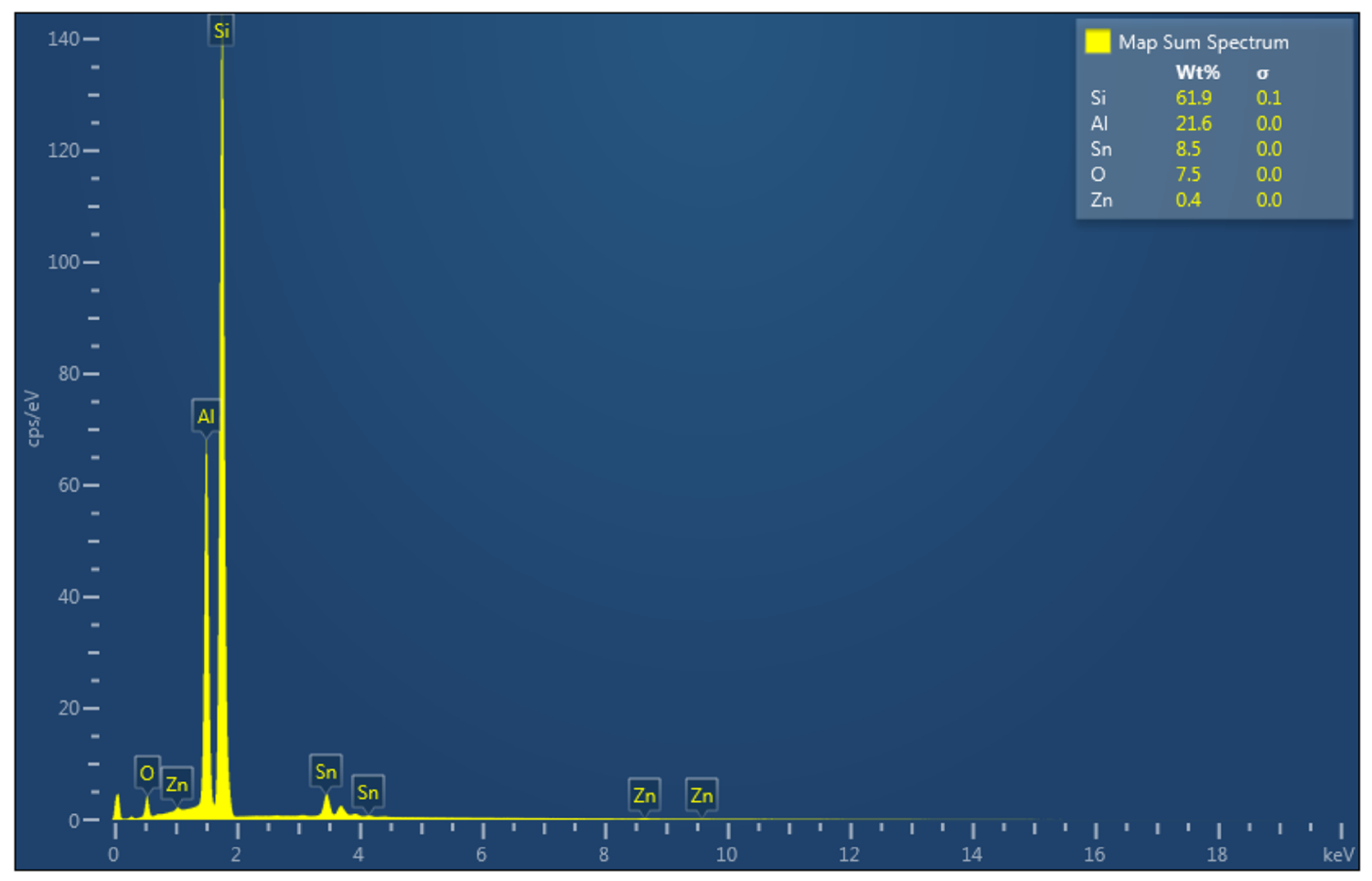

Figure 7. EDS spectra. 
figure 5. The larger active area of the detector offers a larger junction capacitance resulting in greater detector noise. This is one of the main reasons because of which no separation is observed between the Am-peak and the Pu-peak. These bigger area detectors are useful for alpha counting. To have a better morphological picture of the $\mathrm{SnO}_{2}$ layer, the scanning electron microscopy (SEM) images of its surface were taken at two different scales and they are shown in figure 6. In this figure, the SEM image at a $100 \mathrm{~nm}$ scale is presented in the inset and the main image corresponds to a $200 \mathrm{~nm}$ scale. The SEM micrograph shows a closely packed distribution of $\mathrm{SnO}_{2}$ nano-crystals with a size of $\sim 50 \mathrm{~nm}$. The energy dispersive $\mathrm{X}$-ray spectroscopy (EDS) spectrum is also measured in three different locations on the surface of the $\mathrm{SnO}_{2}$ layer and the most detailed one is shown in figure 7. The EDS spectrum shows mainly the peaks corresponding to the $\mathrm{Sn}$ and $\mathrm{O}$ in the $\mathrm{SnO}_{2}$ layer. It is also observed that zinc is present as a metallic impurity in very little quantity which might be responsible for the higher reverse leakage current.

\section{Conclusion}

SSB detectors were fabricated with thin tin oxide passivation layers which can be deposited easily at lower temperatures and also in ambient air. The $\mathrm{SnO}_{2}$ layer on the SSB detector surface was deposited by using the L-B technique. Reverse $I-V$ characteristics of the detector were studied for a period of two and half years. Although the detector showed a lower leakage current compared to a detector without any passivation, the leakage current was increased by about one order after two and a half years. With this cost-effective and easier method of depositing passivation layers, these detectors can find applications in alpha radiation counting.

\section{Acknowledgements}

The authors are deeply thankful to Dr R Tewari of MSD, BARC for the valuable support in carrying out the SEM measurements. The authors also express their sincere thanks to M/s Allied Publishers Pvt. Ltd. (New Delhi, India) for granting copyright permission to reuse a figure from an article from the Proceedings of DAE Solid State Physics Symposium, vol 46, p 293 (2003) for comparison purposes.

\section{References}

[1] Zasadzinski J A, Viswanathan R, Madsen L, Garnaes J and Schwartz D K 1994 Science 2631726

[2] Kemmer J 1984 Nucl. Instrum. Methods 22689

[3] Burger P and Beroud Y 1984 Nucl. Instrum. Methods 22645

[4] Dearnaley G and Whitehead A B 1961 Nucl. Instrum. Methods $\mathbf{1 2} 205$

[5] Fox R J and Borkowski C J 1962 IRE Trans. NS 9213

[6] Andersson G and Zausig B 1966 Nucl. Instrum. Methods 40 277

[7] Stojanovic M, Osmokrovic P, Boreli F, Novkovic D and Webb R 1997 Thin Solid Films 296181

[8] Choudhury S, Betty C A and Girija K G 2008 Thin Solid Films $\mathbf{5 1 7} 923$

[9] Choudhury S, Betty C A and Girija K G 2011 BARC Newsl. 318 39

[10] Betty C A, Choudhury S and Girija K G 2014 Sens. Actuators B 193484

[11] Ray A, Sawant S, Choudhury S and Yakhmi J V 2003 Proc. DAE Solid State Phys. Symp. $\mathbf{4 6} 293$ 